\title{
Genome-wide analysis and expression profiles of PdeMYB transcription factors in colored-leaf poplar (Populus deltoids)
}

\author{
Weibing Zhuang ${ }^{*}$, Xiaochun Shu, Xinya Lu, Tao Wang, Fengjiao Zhang, Ning Wang and Zhong Wang ${ }^{*}$
}

\begin{abstract}
Background: $M Y B$ transcription factors, comprising one of the largest transcription factor families in plants, play many roles in secondary metabolism, especially in anthocyanin biosynthesis. However, the functions of the PdeMYB transcription factor in colored-leaf poplar remain elusive.

Results: In the present study, genome-wide characterization of the PdeMYB genes in colored-leaf poplar (Populus deltoids) was conducted. A total of 302 PdeMYB transcription factors were identified, including 183 R2R3-MYB, five R1R2R3-MYB, one 4R-MYB, and 113 1R-MYB transcription factor genes. Genomic localization and paralogs of PdeMYB genes mapped 289 genes on 19 chromosomes, with collinearity relationships among genes. The conserved domain, gene structure, and evolutionary relationships of the PdeMYB genes were also established and analyzed. The expression levels of PdeMYB genes were obtained from previous data in green leaf poplar (L2025) and colored leaf poplar (QHP) as well as our own qRT-PCR analysis data in green leaf poplar (L2025) and colored leaf poplar (CHP), which provide valuable clues for further functional characterization of PdeMYB genes.
\end{abstract}

Conclusions: The above results provide not only comprehensive insights into the structure and functions of PdeMYB genes but also provide candidate genes for the future improvement of leaf colorization in Populus deltoids.

Keywords: Genome-wide, PdeMYB transcription factors, Populus deltoids, Expression profiles, Anthocyanin biosynthesis

\section{Background}

Transcription factors play important roles in the regulation of gene expression, which can control the rate of transcription initiation of target genes. $M Y B$ transcription factors, one of the largest and best-characterized transcription factor gene families in plants, are widely distributed in all eukaryotic organisms, including animals, plants, and fungi $[1,2]$, which can be involved in various types of plant growth and development.

\footnotetext{
*Correspondence: weibingzhuangnj@sina.com; wangzhong@cnbg.net Jiangsu Key Laboratory for the Research and Utilization of Plant Resources, Institute of Botany, Jiangsu Province and Chinese Academy of Sciences (Nanjing Botanical Garden Mem. Sun Yat-Sen), Nanjing 210014, China
}

Most transcription factors are structurally classified into different families based on their domain diversity $[3,4]$. Similarly, MYB transcription factors can be classified into four types according to the number of MYB domains: 1R-MYB, R2R3-MYB, R1R2R3-MYB, and 4R-MYB [5-8]. Among these $M Y B$ transcription factors, the R2R3-MYB group is the largest transcription factor subfamily in plants $[8,9]$. As the R2R3-MYB transcription factor is the largest transcription factor subfamily, genome identification of R2R3-MYB transcription factors has been conducted in several sequenced plants. There are 126 R2R3-MYB transcription factors in Arabidopsis thaliana [10], 108 in grape [11], 100 in sweet orange [12], 222 in apple [13] and 192 genes in Populus trichocarpa [14].

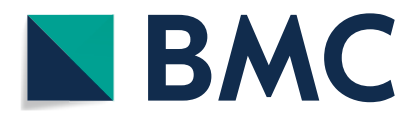

(C) The Author(s) 2021. Open Access This article is licensed under a Creative Commons Attribution 4.0 International License, which permits use, sharing, adaptation, distribution and reproduction in any medium or format, as long as you give appropriate credit to the original author(s) and the source, provide a link to the Creative Commons licence, and indicate if changes were made. The images or other third party material in this article are included in the article's Creative Commons licence, unless indicated otherwise in a credit line to the material. If material is not included in the article's Creative Commons licence and your intended use is not permitted by statutory regulation or exceeds the permitted use, you will need to obtain permission directly from the copyright holder. To view a copy of this licence, visit http://creativecommons.org/licenses/by/4.0/. The Creative Commons Public Domain Dedication waiver (http://creativeco mmons.org/publicdomain/zero/1.0/) applies to the data made available in this article, unless otherwise stated in a credit line to the data. 
Most R2R3-MYB proteins among different species are largely conserved, and they can cluster into the same subgroups according to their sequence similarity. However, divergence between them also exists. A comparative analysis of R2R3-MYB genes among different plant species revealed that this transcription factor gene family has undergone extensive expansion during evolution. The expansion of the R2R3-MYB family in plants fits well with the observation that many R2R3MYB members participate in various biological processes and plant-specific processes [15].

More and more R2R3-MYB transcription factors have been functionally identified since the first identification of the plant $M Y B$ gene $C 1$ from Zea mays [16]. The R2R3-MYB transcription factors are mainly involved in stamen and pollen maturation [17], trachoma initiation [18], root formation and development $[19,20]$, hormone signal transduction [21], embryogenesis, and stress [22-24]. Recently, the functions of R2R3-MYB transcription factors in the regulation of secondary metabolism (particularly anthocyanin biosynthesis and metabolism) have been reported in many species, including apple [25], grape [26], litchi [27] and strawberry [28]. Based on phylogenetic analysis, the R2R3-MYB transcription factors associated with anthocyanin biosynthesis in different species typically belong to the same subgroup. In $P$. trichocarpa, the anthocyanin content of male catkins is higher, and the transcript levels of R2R3-MYB transcription factors, including PtrMYB116, PtrMYB117, PtrMYB118, and PtrMYB119, in the male catkins of P. trichocarpa are also higher. Another R2R3-MYB transcription factor, PtrMYB120, could play a role in vegetative tissues by evoking anthocyanin biosynthesis to protect them from the deleterious effects of UV light [14]. Transgenic poplars overexpressing PtrMYB119, PtrMYB120, or PdeMYB118 show an elevated accumulation of anthocyanins throughout the plant [29, 30]. Although some R2R3-MYB transcription factors associated with anthocyanin biosynthesis have been identified, many more MYB transcription factors associated with anthocyanin biosynthesis are required to obtain new coloredleaf tree species.

With the development of the social economy, the roles of colored-leaf trees in urban beautification are increasing. Leaf color formation is very complicated, and the distribution and concentration of anthocyanins play important roles in the formation of leaf color. Anthocyanin accumulation is controlled through the coordinated expression of genes encoding the anthocyanin biosynthetic pathway enzymes, and R2R3-MYB transcription factors are considered crucial in the regulation of anthocyanin synthesis.
In the current study, a comprehensive investigation of $P d e M Y B$ transcription factors in colored-leaf poplar (Populus deltoids) was conducted, and the analysis of phylogenetic relationships, sequence features, gene duplication, chromosome distribution, and motif recognition was performed. In addition, a comprehensive expression analysis of $P d e M Y B$ genes in green leaf poplar (L2025) and colored leaf poplar (QHP) using previous RNA-seq data was performed. The expression levels of candidate PdeMYB genes in green leaf poplar (L2025) and colored leaf poplar (CHP) were also evaluated by qRT-PCR analysis. These findings should not only provide a characterization of the $P d e M Y B$ gene superfamily but also provide valuable information for further functional elucidation of these genes in colored-leaf poplar, which is useful for generating colored-leaf tree species by genetic engineering.

\section{Results}

\section{Genome-wide identification of the PdeMYB gene family in P. deltoids}

A total of 302 PdeMYB genes were identified based on the complete genome sequences of $P$. deltoids. The length of amino acids for the identified $P d e M Y B$ proteins ranged from 51 bp (PdeMYB190) to 1717 bp (PdeMYB289), with an average of $342.8 \mathrm{bp}$ (Additional file 1). The molecular weight of the identified $P d e M Y B$ proteins ranged from 5.6084 kDa (PdeMYB190) to $188.4509 \mathrm{kDa}$ (Pde$M Y B 289)$, and the predicted isoelectric points of these ranged from 4.08 (PdeMYB223) to 10.98 (PdeMYB298). In addition, the subcellular localization of the identified PdeMYB proteins was also predicted, and 208 of 302 (approximately 69\%) PdeMYB proteins were localized in the nucleus (Additional file 1).

In plants, MYB proteins are characterized by a highly conserved MYB domain at the N-terminus, which contains one to four imperfect repeats, and can be classified into four major subfamilies: 1R-like MYB, R2R3-MYB, R1R2R3-MYB, and 4R-MYB. In our study, a total of 302 $P d e M Y B$ transcription factors were identified, including 183 R2R3-MYB, five R1R2R3-MYB, one 4R-MYB, and 113 1R-MYB.

\section{Phylogenetic analysis of the PdeMYB gene family between $P$. deltoids and Arabidopsis}

An unrooted phylogenetic tree among the R2R3-MYB, R1R2R3-MYB, and 4R-MYB genes of $P$. deltoids, rice, and Arabidopsis was constructed using the neighbor-joining method with MEGA 7.0 (Fig. 1). The PdeMYB genes of $P$. deltoids were divided into 10 groups (I to X) according to their sequences. Each group contained a different number of PdeMYB genes. Group III contained 50 $P d e M Y B$ genes, which was the largest number in the 


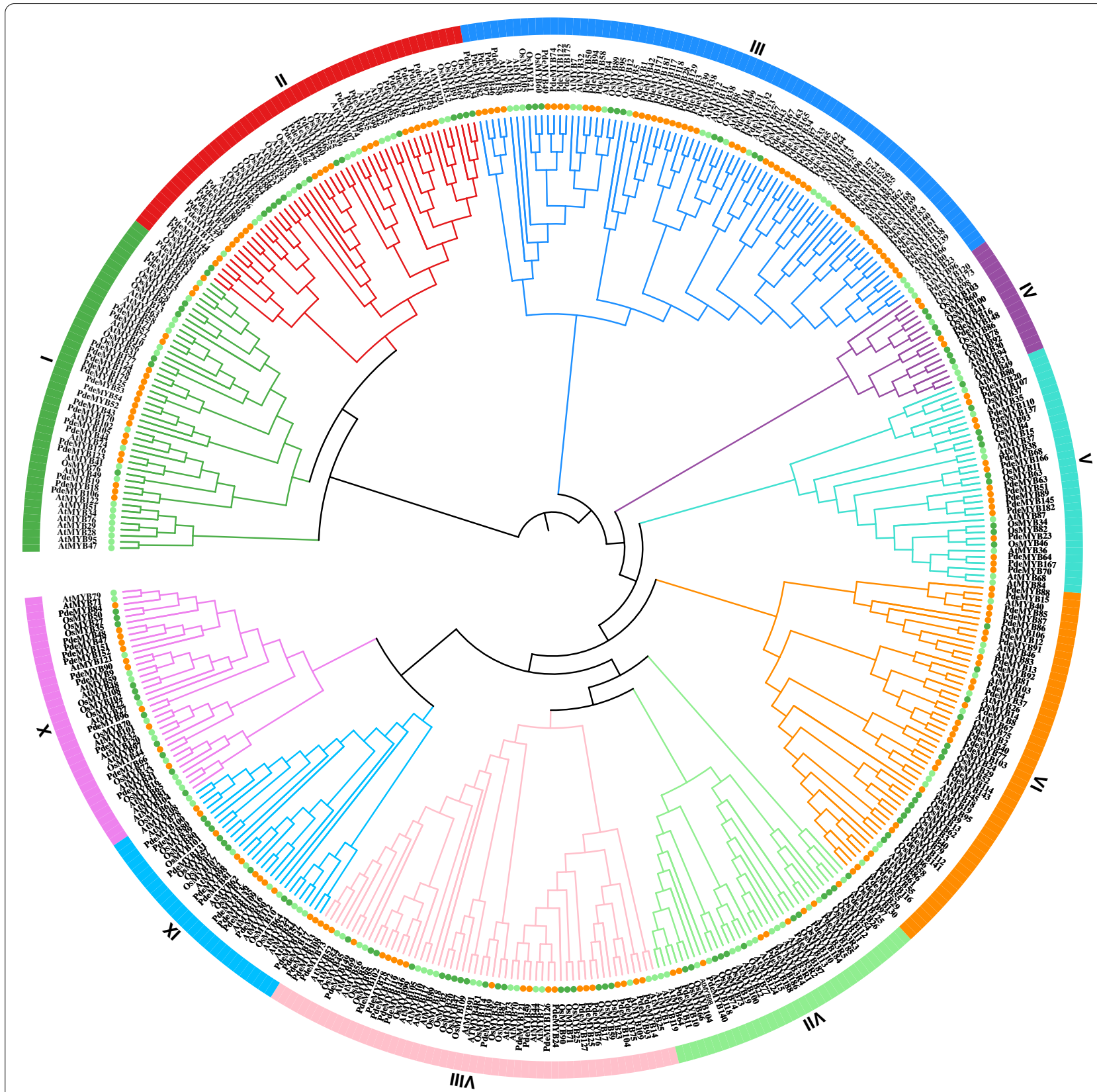

Fig. 1 Phylogenetic analysis of R2R3-MYB, R1R2R3-MYB and 4R-MYB gene families in Arabidopsis, rice and P. deltoids. An un-rooted phylogenetic tree of MYB genes in Arabidopsis, rice and P. deltoids was constructed using the neighbor-joining method in MEGA 7.0 software with a bootstrap test (replicated 1000 times). The MYB gene families in Arabidopsis, rice and $P$. deltoids were marked light green, dark green and yellow, respectively

10 groups, while group IV contained 4 PdeMYB genes, which was the smallest number in the 10 groups. Except group IV, VII, and X, the other groups of $P$. deltoids possessed much more MYB gene members than these of Arabidopsis and rice in the seven out of ten groups. Each group could be further divided into several subgroups. Some subgroups just included $P$. deltoids $M Y B$ genes but no AtMYB and $O s M Y B$ gene, indicating that these genes may have occurred in $P$. deltoids during evolutionary process. While some subgroups just contained $A t M Y B$ or $O s M Y B$ genes with no $P$. deltoids $M Y B$ genes, suggesting that some evolutionary changes occurred in the genome - the MYB genes could have been either acquired in Arabidopsis or rice during evolution or lost in P. deltoids. The gain and loss of species-specific MYB genes could have resulted in functional divergence. 


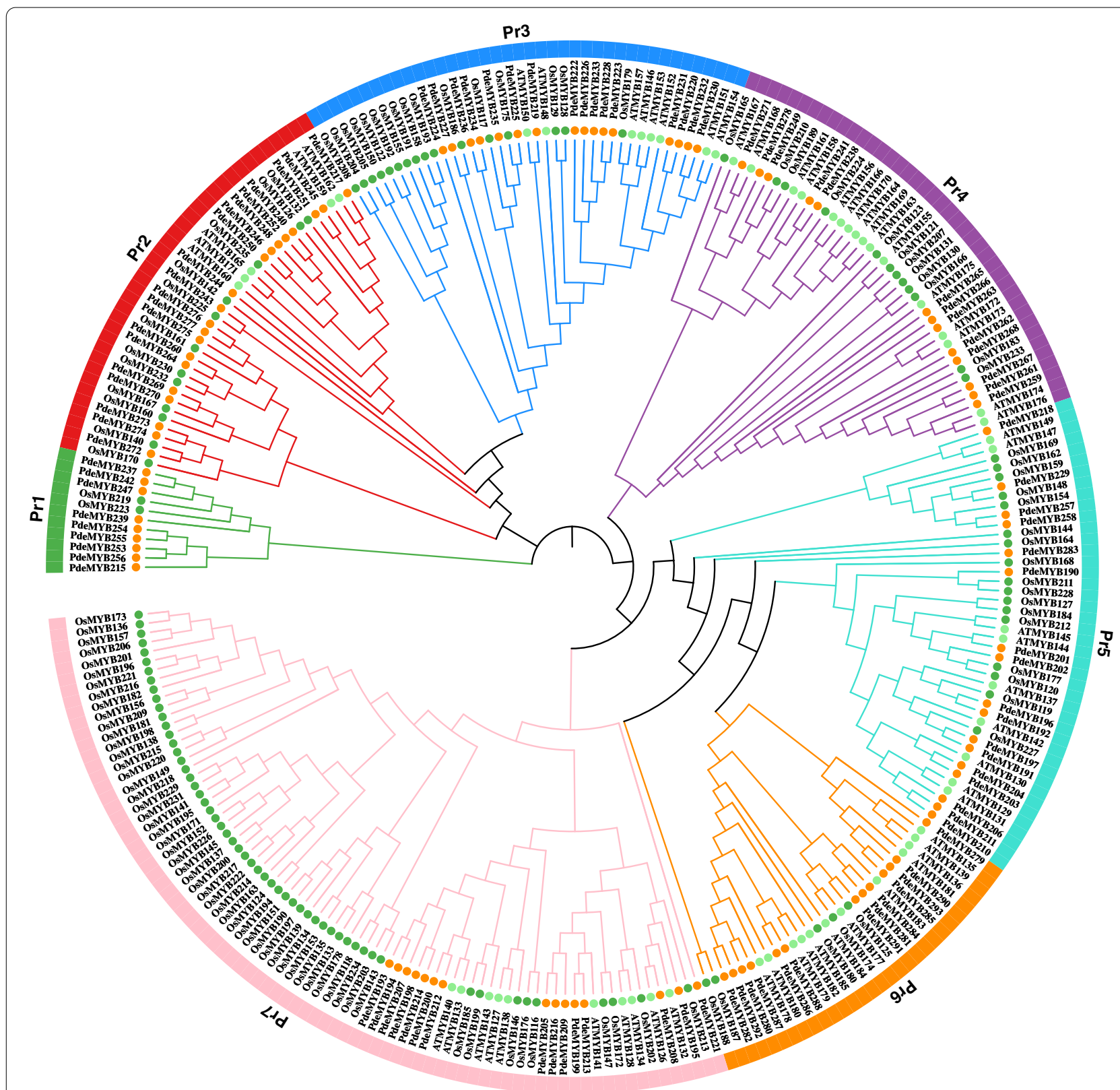

Fig. 2 Phylogenetic analysis of 1R-MYB gene families in Arabidopsis, rice and P. deltoids. An un-rooted phylogenetic tree of MYB gene family among Arabidopsis, rice and $P$. deltoids was constructed using the neighbor-joining method in MEGA 7.0 software with a bootstrap test (replicated 1000 times). The MYB gene families in Arabidopsis, rice and P. deltoids were marked light green, dark green and yellow, respectively

The phylogenetic tree of 1R-MYB genes in Arabidopsis, rice, and $P$. deltoids was constructed using the neighbor-joining method with MEGA 7.0 (Fig. 2). The $P$ deMYB genes in $P$. deltoids were divided into seven groups based on their sequences. Most groups contained the 1R-MYB genes of $P$. deltoids, rice and Arabidopsis, while $\operatorname{Pr} 1$ group just contained the 1R-MYB genes of $P$. deltoids and rice. Pr2 contained 20 Pde$M Y B$ genes, which was the largest number in the seven groups, while Group Pr1 contained 9 PdeMYB genes, which was the smallest number in the seven groups. Except group Pr4 and Pr7, the other groups of $P$. deltoids possessed much more $M Y B$ gene members than these of Arabidopsis and rice in the five out of seven groups. In Pr1 group, one of the subgroups contained all 1R-MYB genes of $P$. deltoids including PdeMYB215, PdeMYB258, PdeMYB253, PdeMYB255 and PdeMYB254. In Pr6 group, one of the 
subgroups contained all $1 \mathrm{R}-\mathrm{MYB}$ genes of rice, such as OsMYB173, OsMYB136, OsMYB157, OsMYB206 and so on. In Pr4 group, one of the subgroups contained all 1R-MYB genes of Arabidopsis including AtMYB156, AtMYB163, AtMYB164, AtMYB166, AtMYB169, and AtMYB170. There are much more MYB gene members of rice in Pr7 than these of $P$. deltoids and Arabidopsis, which indicated that these $M Y B$ genes could be either acquired in rice during evolution or lost in $P$. deltoids and Arabidopsis. The above results also indicated the differences during the evolutionary process of $1 \mathrm{R}-\mathrm{MYB}$ genes between P. deltoids and Arabidopsis.

\section{Conserved gene structure and protein motif analysis of the PdeMYB gene family in P. deltoids}

To better understand the structural diversity and motif composition of PdeMYB genes, the intron-exon structure pattern was analyzed and visualized using the Gene Structure Display Server 2.0 (Fig. 3). A total of 292 PdeMYB genes possessed exons varying from 1 to 10, which accounted for $97 \%$ of the total PdeMYB genes. Sixteen $P d e M Y B$ genes lacked introns and had only one exon, including PdeMYB14, PdeMYB24, PdeMYB25, PdeMYB59, PdeMYB76, PdeMYB121, PdeMYB126, PdeMYB127, PdeMYB190, PdeMYB229, PdeMYB248, PdeMYB264, PdeMYB266, PdeMYB267, PdeMYB294, and PdeMYB295. The majority (164 of 302) of the Pde$M Y B s$ had typical splicing, with three exons and two introns. PdeMYB256 and PdeMYB257 contained 19 exons and 18 introns, which was the greatest number of exons in the total PdeMYB genes. Moreover, the number of introns in the $M Y B$ genes appeared to be limited, as most $P \operatorname{deMYB}$ genes (75\%) had no more than two introns. Phylogenetic analysis of the PdeMYB gene family was performed according to the intron number and exon length. As the position(s) of the intron(s) were fully conserved, genes in the same subgroups had similar intron patterns, such as in PdeMYB238/PdeMYB246 and PdeMYB113/PdeMYB142 (Fig. 3).

As shown in Fig. 4, six conserved motifs were predicted to further reveal the diversification of PdeMYB genes in $P$. deltoids. In most cases, a motif is repeated only once or twice, and one gene has only one motif repeated. However, only a few special cases exist. PdeMYB71, PdeMYB194, PdeMYB170, and PdeMYB83 contained two repeated motifs, and each motif was repeated twice. PdeMYB6 contained three repeated motifs: motif 3 repeated three times, motif 4 repeated twice, and motif 6 repeated four times. PdeMYB57 also contained three repeated motifs, and each motif was repeated twice. PdeMYB21 contained four repeated motifs: motif 2 repeated twice, motif 3 repeated twice, motif 4 repeated twice, and motif 5 repeated twice.

\section{Chromosomal location and duplication events of PdeMYBs in $P$. deltoids}

To determine the genomic distribution of the PdeMYB gene family in $P$. deltoids, the chromosomal locations of $P d e M Y B s$ were evaluated according to their genomic sequences. There are 19 chromosomes in the P. deltoids genome, and each of the $19 \mathrm{P}$. deltoids chromosomes contained PdeMYB genes (Fig. 5). Although PdeMYB genes were distributed on all chromosomes in P. deltoids, their distribution on each chromosome seemed to be uneven. In the present results, there were 289 PdeMYB genes assigned to 19 chromosomes: 32 PdeMYBs were present on chromosome 1; 23 on chromosome 2; 20 on chromosomes 4 and 15; 19 on chromosomes 6 and 8; 18 on chromosome 17; 16 on chromosomes 3 and 10; 14 on chromosome $5 ; 13$ on chromosomes 12,13 , and 14; 12 on chromosomes 9 and 18; 11 on chromosome 19; 9 on chromosome 9; 5 on chromosome 16; and 4 on chromosome 11. Chromosome 1 had the highest number of PdeMYB genes (32), followed by chromosome 2 (23). Chromosome 11 had the least number of PdeMYB genes (4). In addition, 13 PdeMYB genes belonged to the scaffold.

Gene duplication, especially tandem and segmental duplication events, contributes greatly to the diversity and evolution of gene families. In the present study, there were 59 duplicated PdeMYB gene pairs in the $P$. deltoids genome, and most of them belonged to the segmental duplication or whole genome duplication event. A total of 56 pairs of $P d e M Y B$ genes were identified as whole genome duplication or segmental duplications, including 53 duplication events between different chromosomes as well as three duplication events within the same chromosome (PdeMYB10/PdeMYB11, PdeMYB12/PdeMYB13, and PdeMYB47/PdeMYB48), while three pairs of Pde$M Y B s$ were found as tandem repeats in P. deltoids, including PdeMYB156/PdeMYB157, PdeMYB53/PdeMYB54, and PdeMYB155/ PdeMYB156. Interestingly, genes such as PdeMYB156, PdeMYB277, PdeMYB162, and Pde$M Y B 47$ occurred in more than two gene pairs (Additional file 2).

To evaluate the selection of the duplicated PdeMYB gene pairs, the non-synonymous to synonymous substitution ratios $(\mathrm{Ka} / \mathrm{Ks})$ were calculated according to the whole genome analysis of gene duplications. When the $\mathrm{Ka} / \mathrm{Ks}$ ratio is greater than one, the identified genes are under positive selection; when the $\mathrm{Ka} / \mathrm{Ks}$ ratio is one, the identified genes are under neutral selection; when the $\mathrm{Ka} /$ Ks ratio is less than one, the identified genes are under 


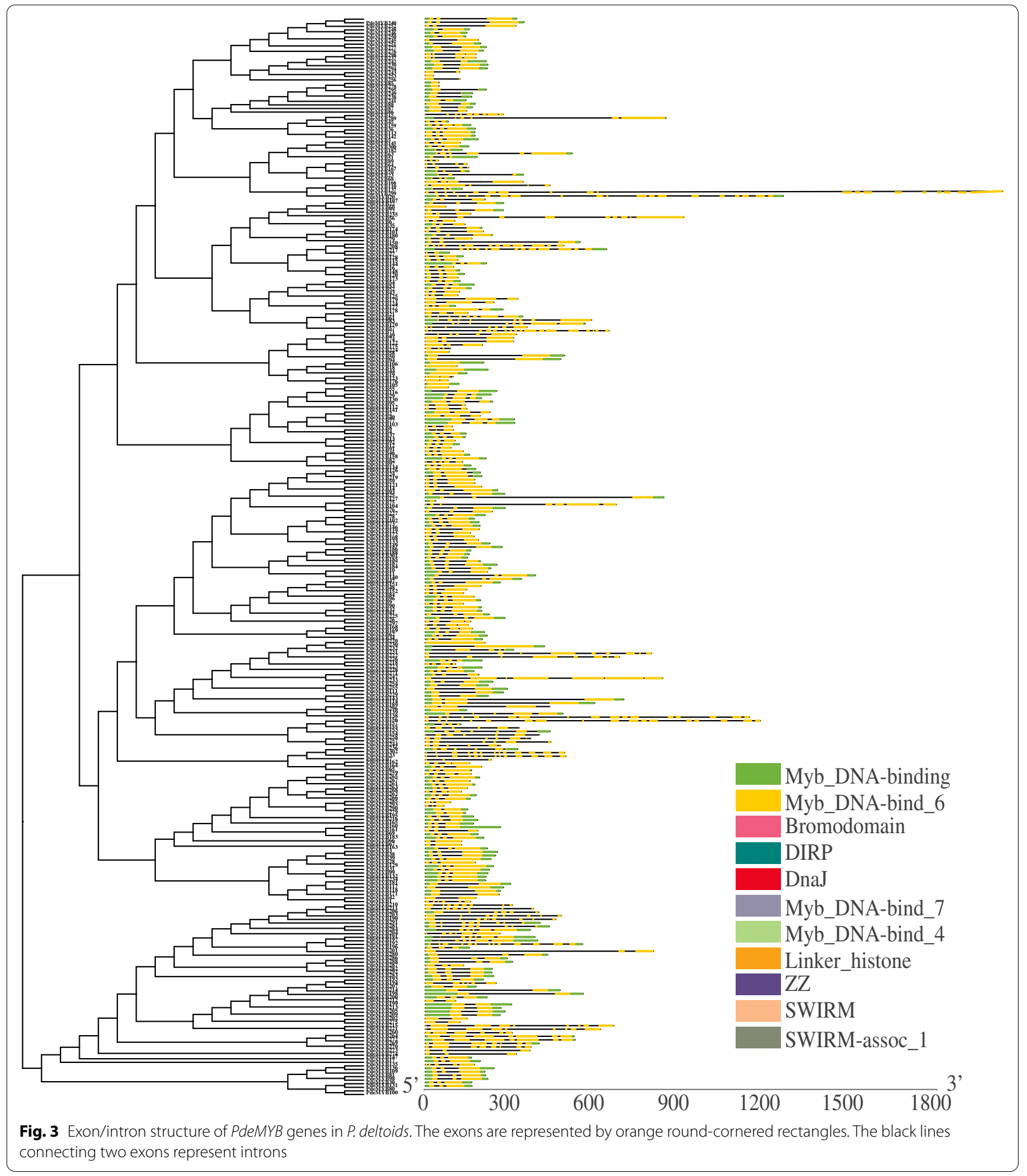

negative purifying selection. In the present study, there were 57 PdeMYB gene duplicated pairs, the $\mathrm{Ka} / \mathrm{Ks}$ ratios of which were less than one, indicating that these genes are under negative purifying selection and contribute largely to the maintenance of function in the $P \operatorname{deMYB}$ gene family of $P$. deltoids. There were two PdeMYB gene duplicated pairs, the $\mathrm{Ka} / \mathrm{Ks}$ ratios of which were more than one, indicating that these genes have likely 


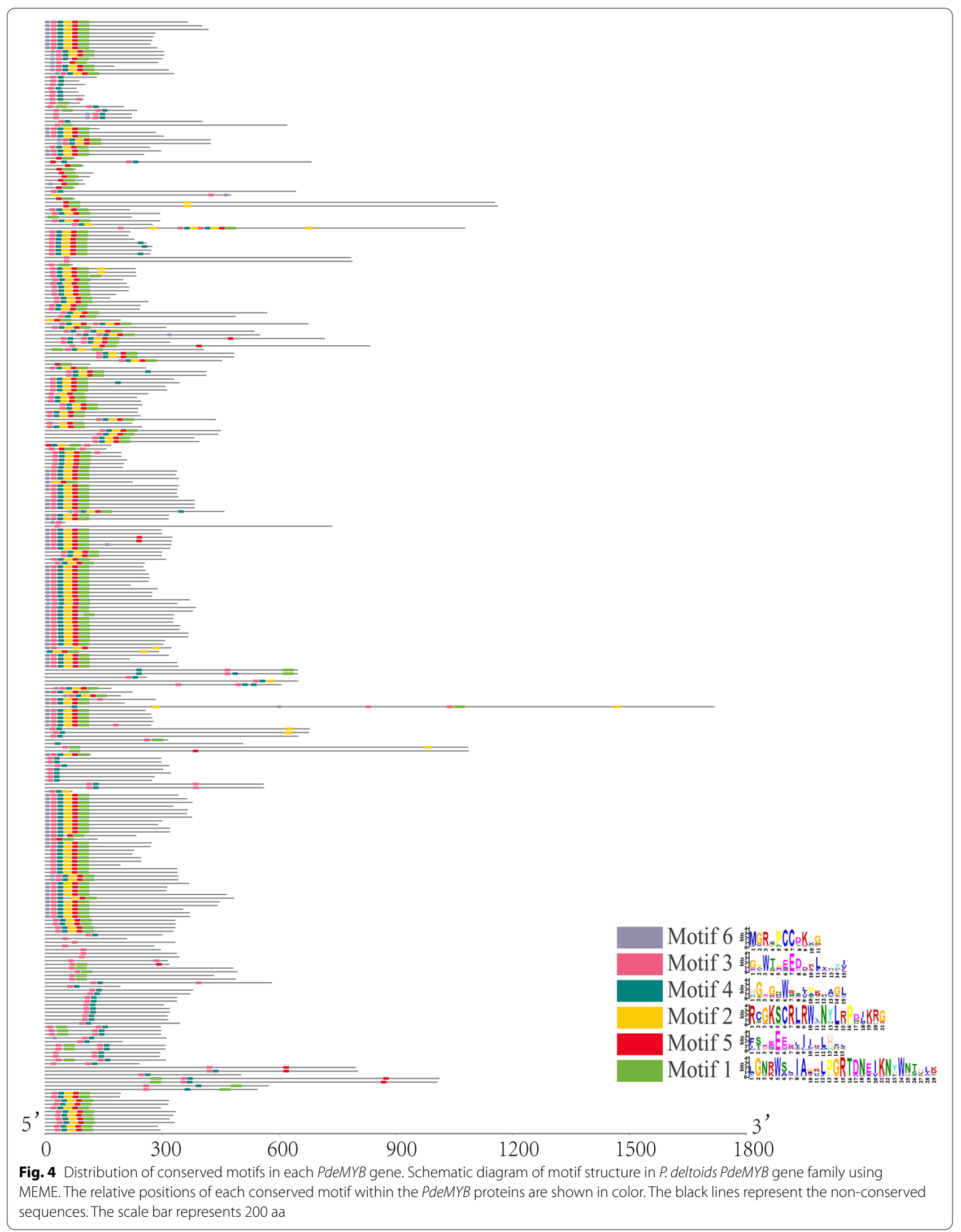




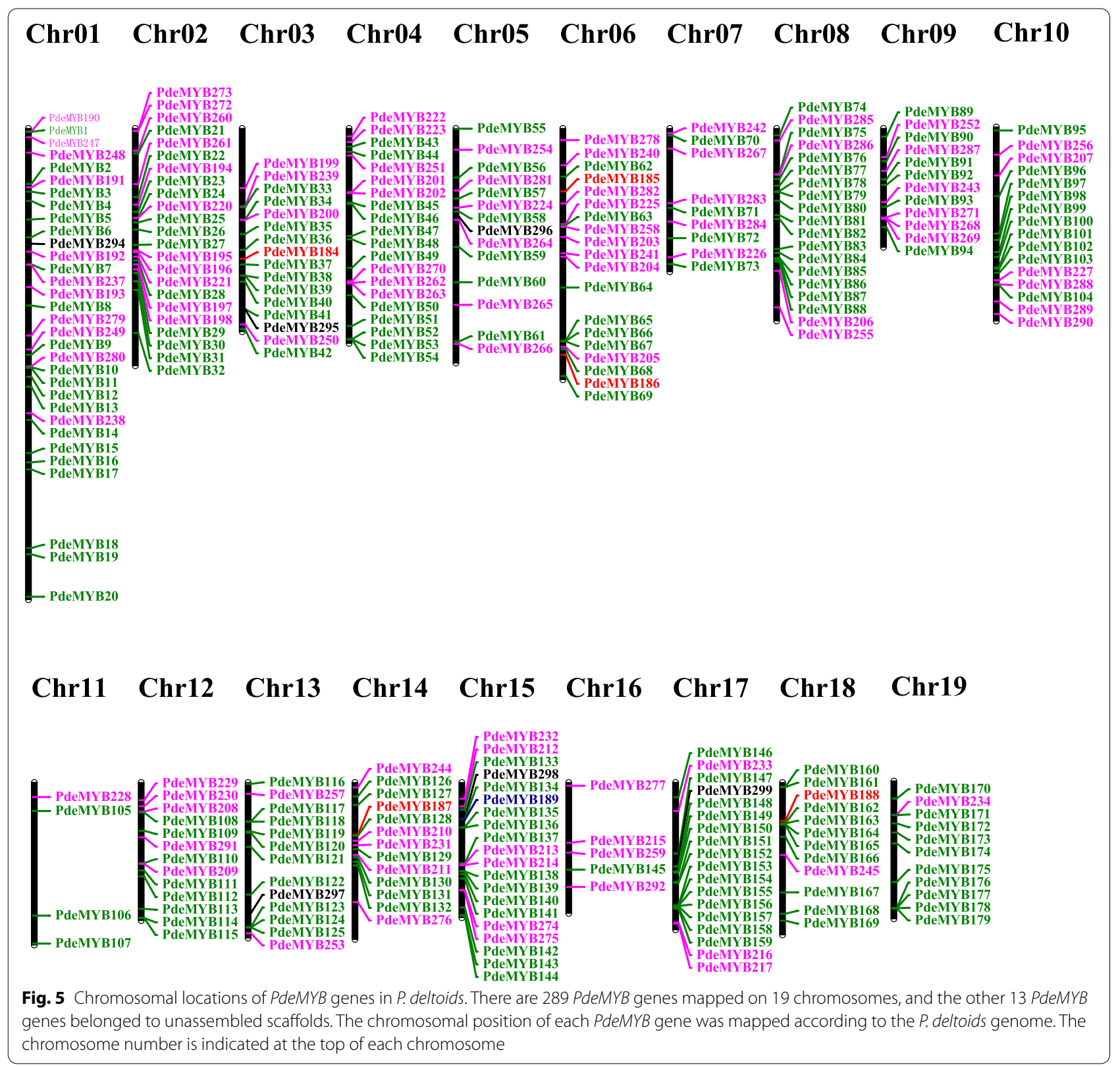

experienced positive selection. Ks was used as a proxy for time to estimate the dates of the duplication event. The segmental duplicated events in $P$. deltoids appeared to have occurred from $0.001264253(\mathrm{Ks}=3.79 \mathrm{E}-05)$ to 50.944 mya $(\mathrm{Ks}=1.52832)$. The $\mathrm{Ks}$ of tandem duplication of PdeMYB genes occurred from 1.823416667 to 7.34143333 mya (Additional file 2).

To evaluate the possible relationship between the $P d e M Y B$ genes and potential duplication events, the collinearity of the PdeMYB gene family in $P$. deltoids was identified using the BLASTP and MCScanX methods. A total of 56 segmental duplication events with
$102 P d e M Y B$ genes were identified in the $P$. deltoids genome (Fig. 6). PdeMYB genes were located within synteny blocks on all chromosomes. Intrachromosomal duplication was also observed in the $P$. deltoids genome.

To further evaluate the potential evolutionary mechanisms of the PdeMYB gene family in P. deltoids, two comparative syntenic maps were constructed between P. deltoids, Arabidopsis, and rice (Fig. 7). There were 60 collinear MYB gene pairs between P. deltoids and Arabidopsis and 77 orthologs between $P$. deltoids and rice. The details of the collinear $M Y B$ gene pairs were provided in Additional files 3 and 4. The number of orthologous 
Zhuang et al. BMC Plant Biol

(2021) 21:432

Page 9 of 16

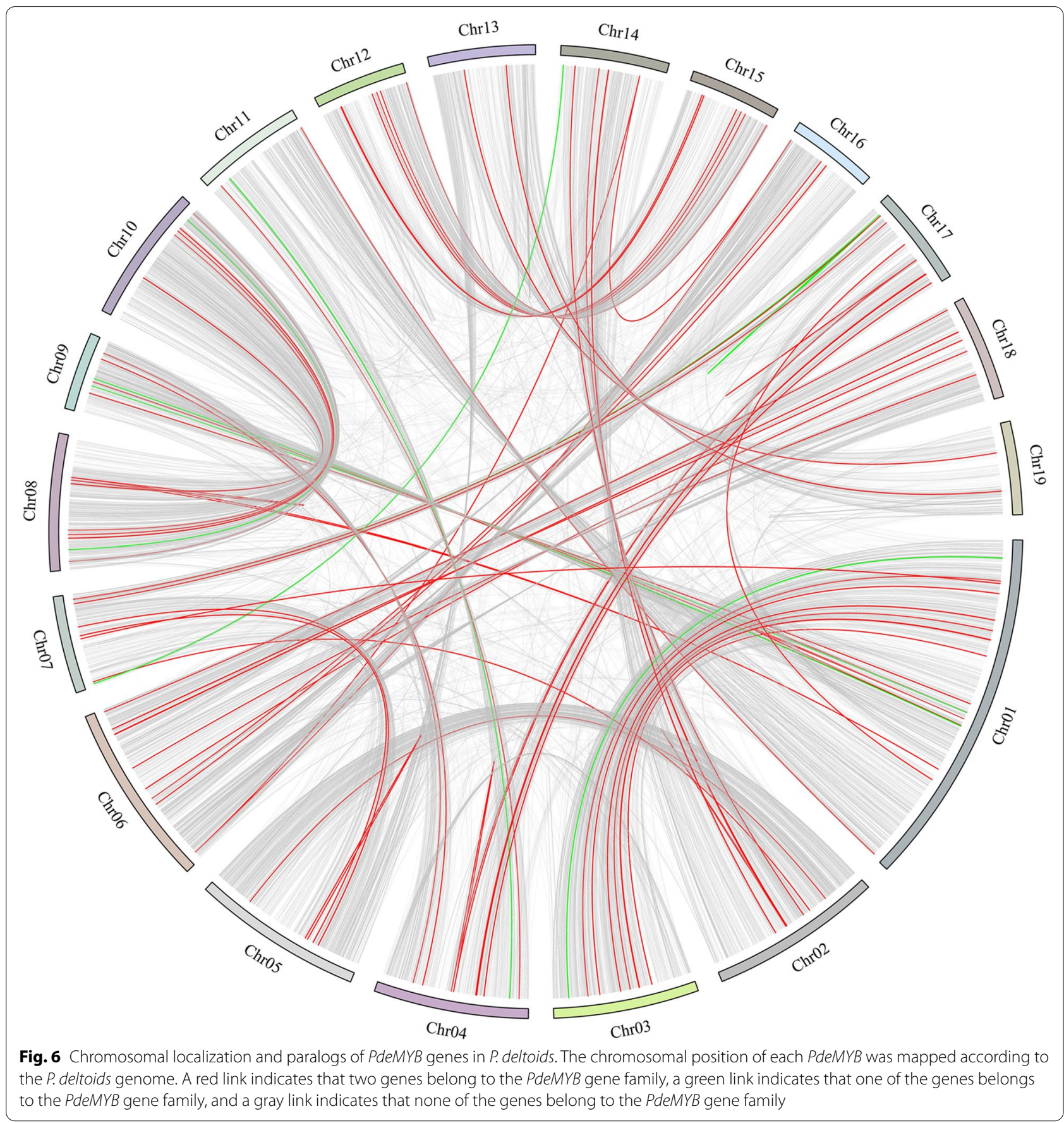

events of $P d e M Y B-O s M Y B$ was much greater than that of $P \operatorname{deMYB}-A t M Y B$ as there were much bigger genome size and much more chromosome numbers.

Expression profile of the PdeMYB genes in QHP and L2025 by RNA-seq

To evaluate the expression pattern of $P d e M Y B$ genes in colored-leaf poplar, the expression profiles of PdeMYB genes in the leaves and buds of QHP and L2025 were screened from the previous data. The expression levels of candidate PdeMYB genes in the leaves of QHP and L2025 were also evaluated (Fig. 8). The expression level of candidate PdeMYB genes in the leaves of QHP was more than 10 times that in L2025 or specifically expressed in the leaves of QHP or L2025, and these candidate $P \operatorname{deMYB}$ genes are shown in Additional 

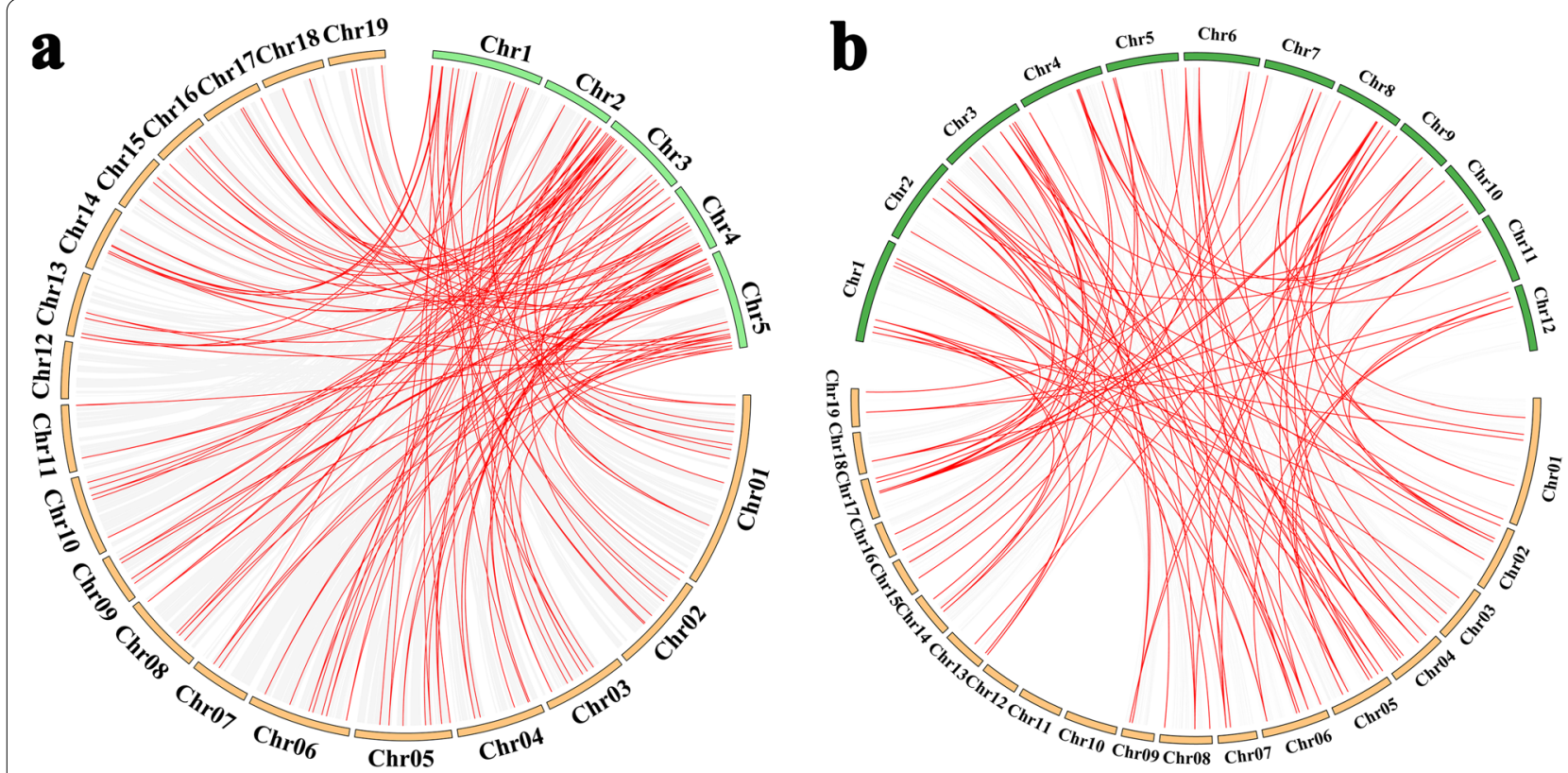

Fig. 7 Gene duplication and synteny analysis of MYB genes between P. deltoids, Arabidopsis, and rice. a Gray link in the background indicates the collinear blocks within P. deltoids and Arabidopsis genomes, while the red link highlights the syntenic MYB gene pairs. b Gray link in the background indicates the collinear blocks within $P$. deltoids and rice genomes, while the red lines highlight the syntenic $M Y B$ gene pairs

file 5. The expression level of PdeMYB154 in the leaves of QHP was 13.28 times higher than that of L2025, and 33.21 times higher for PdeMYB155. Many Pde$M Y B$ transcription factors are specifically expressed in QHP, such as PdeMYB25, PdeMYB27, PdeMYB60, Pde$M Y B 114$, and PdeMYB160, which indicated that these genes might play important roles in the formation of colored leaves in QHP.

\section{Expression analyses of PdeMYB genes in CHP and L2025 by qRT-PCR analysis}

To better explore the expression pattern of $P d e M Y B$ genes in leaf-colored poplar, the candidate $P d e M Y B$ genes were also evaluated in the leaves of $\mathrm{CHP}$ and L2025 (Fig. 9). The expression levels of some PdeMYB genes between CHP and L2025 were not substantially different, such as PdeMYB25, PdeMYB99, and P9de$M Y B 279$, which may not be important in the coloration of CHP. Some PdeMYB genes such as PdeMYB60, PdeMYB70, PdeMYB96, and PdeMYB114, which are specifically expressed in the leaves of $C H P$, may be candidate $P d e M Y B$ genes to further explore the mechanism of leaf coloration in poplar. Other $P d e M Y B$ genes, such as PdeMYB4, PdeMYB37, PdeMYB72, and $P d e M Y B 146$, which are specifically expressed in the leaves of L2025, may also be important candidate genes for studying their functions.

\section{Discussion}

$M Y B$ transcription factors play important roles in secondary metabolism (especially in the anthocyanin pathway), development, signal transduction, and disease resistance, and comprise one of the largest transcription factor families in plants [7]. Many reports have indicated that MYB transcription factor genes can regulate the formation of leaf coloration in plants. Transgenic Arabidopsis overexpressing PAP1/AtMYB75 increases the expression level of structural genes associated with anthocyanin biosynthesis, which contributes to higher anthocyanin accumulation [7, 31, 32]. Tobacco overexpressing IbMYB1a can upregulate the expression levels of several structural genes, such as ANS and DFR 22 , which can promote the accumulation of anthocyanin [33]. Tobacco overexpressing CsMYB6A increases the expression levels of structural genes associated with flavonoid synthesis, such as $3 G T$ and $C H S$, leading to a high accumulation of anthocyanins in the leaves of transgenic tobacco [34]. Transgenic poplar overexpressing MYB6 shows a red color in the young leaves and shoots by increasing their anthocyanin accumulation [35]. PtrRML1, a repressor motif-containing poplar R3 MYB-like transcription factor, the overexpression of which in transgenic Arabidopsis can reduce their anthocyanin content in stems, petioles, and rosette leaves [36]. Overexpression of PtoMYB156, PtrMYB57, MYB182, $M Y B 165$, and $M Y B 194$ separately can also downregulate 


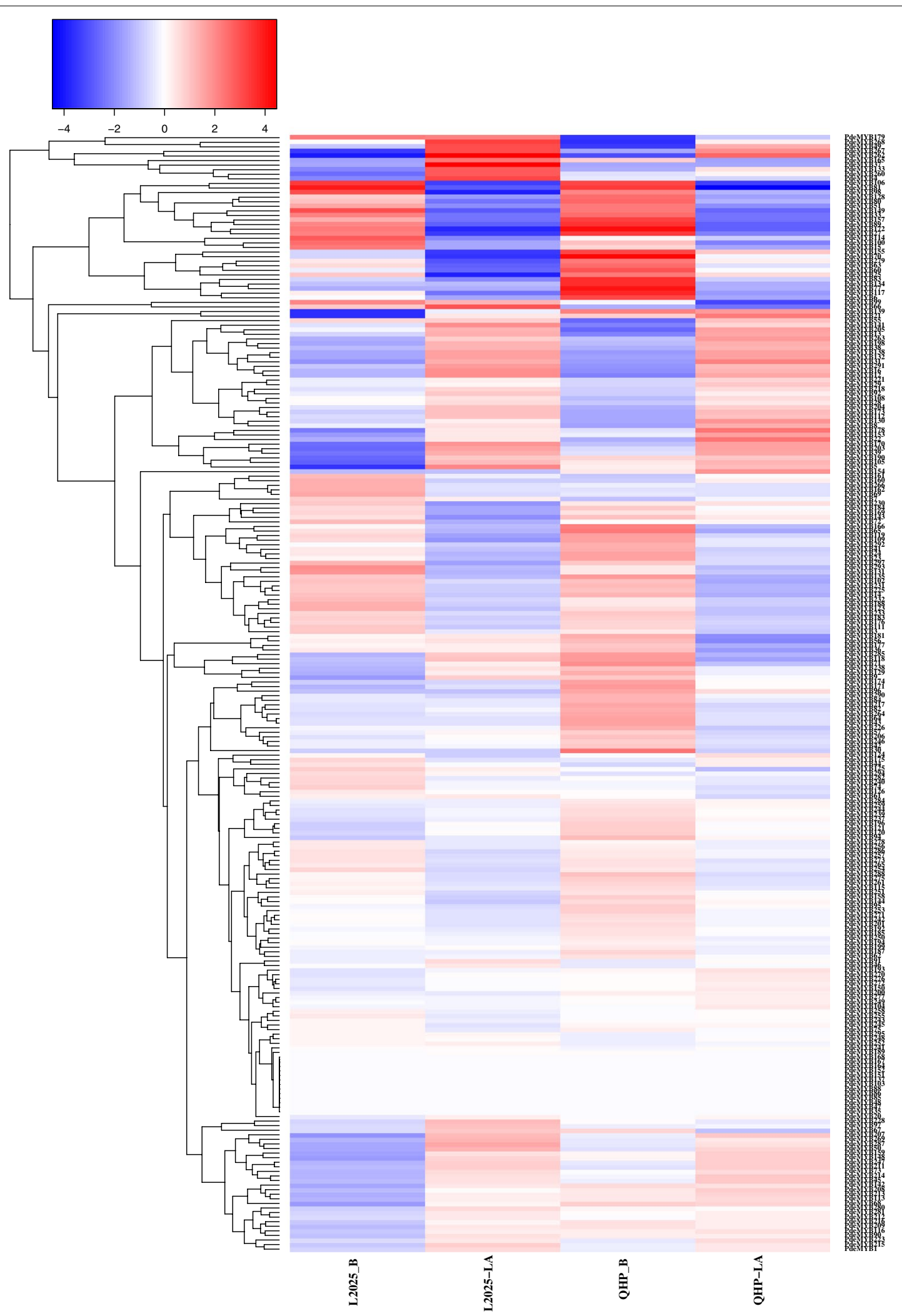

Fig. 8 Expression profile of PdeMYB genes in the leaves and buds of the QHP and L2025. QHP-L, QHP leaf; L2025-L, L2025 leaf; QHP-B, QHP bud; L2025-B, L2025 bud 


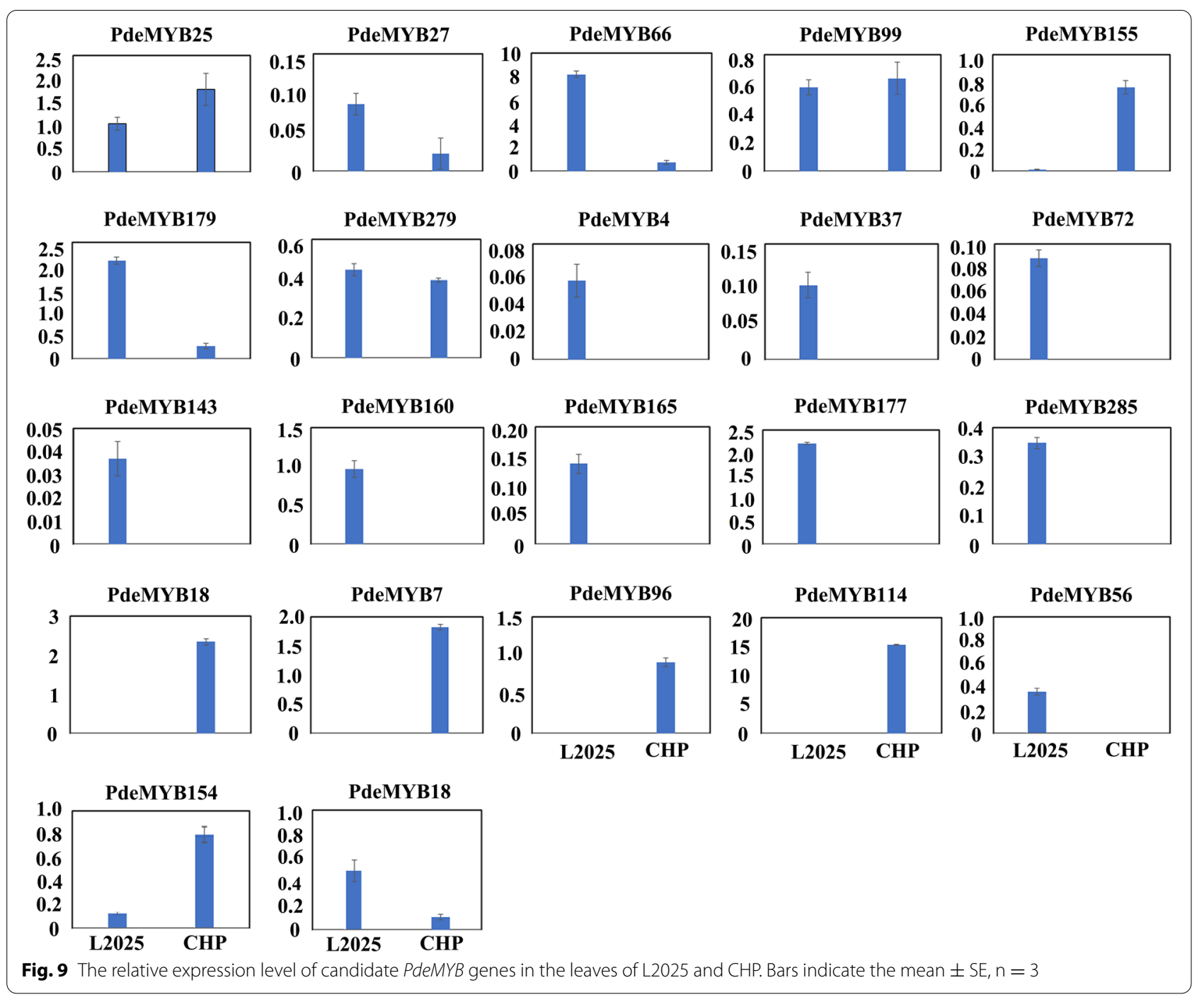

anthocyanin biosynthesis in transgenic poplar [37-40]. Transgenic poplar overexpressing PtrMYB119 and PdeMYB118 separately has higher anthocyanin content in leaves compared with that in wild-type poplar [29, 30]. Although some MYB genes from poplar can regulate the biosynthesis of anthocyanin, many more $M Y B$ genes are needed to regulate the leaf color of poplar. To date, only PtrMYB119 and PdeMYB118 from poplar have been shown to change the leaf color of poplar under normal conditions. Therefore, genome-wide analysis of the $M Y B$ family genes in poplar was characterized, and many more $M Y B$ family genes from poplar associated with anthocyanin biosynthesis need to be identified in the future.

Many $M Y B$ gene families have been identified in different plant species. There are 198, 233, and 197 MYB genes in Arabidopsis, rice, and soybean, respectively [41, 42]. In the present study, 302 PdeMYB transcription factors were identified in the $P$. deltoids genome. Among these,
R2R3-MYBs accounted for $61 \%$ of the identified MYB transcription factors, and MYB-related proteins were the second largest subfamily of MYB proteins, accounting for $37 \%$ in our study, which is similar to the fraction of MYBrelated proteins in rice (40\%) [43]. In P. trichocarpa, there are 192 R2R3-MYB and 5 R1R2R3-MYB [14], which is similar to our results in the $P$. deltoids genome.

Gene duplication plays an important role in gene family expansion in plants [44], either in the form of segmental duplication, tandem duplication, or transposition events [45]. When gene duplication occurs within the same chromosome, tandem duplication occurs. In different chromosomes, this is considered segmental duplication. Both segmental and tandem duplication can lead to the diversification of species, which might also be crucial for increasing the adaptability of plants to different environmental conditions [46]. In the present study, expansion of the R2R3-MYB gene family in P. deltoids resulted 
from both segmental duplication and tandem duplication events, which are similar to those in maize and Gossypium raimondii $[45,47]$. There were three tandem duplications and 56 segmental duplications for PdeMYB genes in $P$. deltoids, which indicated that segmental duplication events were a major cause of the expansion of PdeMYB genes.

It is generally believed that $P \operatorname{deMYB}$ members that fall within certain clades may have common evolutionary origins and conserved functions. Therefore, the putative functions of the $P$. deltoids MYB proteins can be speculated through the functional clades of Arabidopsis MYBs. Phylogenetic analyses and evolutionary relationships of the PdeMYB gene family have been systematically studied among different species, suggesting the conservation and expansion of $P$. deltoids MYBs. In addition, the pattern of gene structures can be used to evaluate the phylogenetic relationships in a gene family. The number of exons in the $302 P d e M Y B$ genes ranged from one to ten, and most of the PdeMYBs had three exons and two introns, which is similar to the results in other plants [9]. Remarkably, the PdeMYB members within the same subfamily shared similar exon/intron patterns [11]. In our study, most of the $P d e M Y B$ genes were disrupted by no more than two introns, which is consistent with previous reports that most MYB-related genes in land plants contain up to two introns [48].

Phylogenetic classification of genes into subfamilies was performed according to the gene structure and domain analysis. In general, members of the same subfamily often share similar sequences, conserved motifs, and even binding partners, and are likely to share similar functions $[49,50]$. Therefore, when there is a lack of sufficient data, functional characterization of unknown proteins is generally carried out based on comparisons with annotated proteins $[13,51]$. In the present study, $P d e M Y B$ genes were clustered into the same group as those in Arabidopsis thaliana, indicating the functional conservation of R2R3-MYBs between species. In Arabidopsis thaliana, MYB75, MYB90, MYB113, and $M Y B 114$ have been reported to be involved in the regulation of anthocyanin biosynthesis $[7,10,51]$. PdeMYB155, PdeMYB157, PdeMYB156, PdeMYB153, and $P d e M Y B 154$ were clustered into the same group as AtMYB75, AtMYB90, AtMYB113, and AtMYB114 in Arabidopsis thaliana, indicating that these genes in poplar might be involved in the synthesis of anthocyanin. In accordance with this, the expression level of PdeMYB154 and PdeMYB155 in the leaves of QHP was much higher than these in L2025 (Fig. 8), and a similar situation occurred in another colored leaf cultivar, CHP (Fig. 9). PdeMYB60 and PdeMYB114 are specially expressed in the leaves of QHP and CHP plants, indicating that they may play important roles in leaf coloration in poplar (Figs. 8 and 9). In contrast to the expression patterns of PdeMYB60 and PdeMYB114 in QHP and CHP, PdeMYB25 was specifically expressed in the leaves of QHP, but there was no significant difference in its expression level between CHP and L2025 (Figs. 8 and 9), which indicated that PdeMYB25 might not be a critical gene during leaf coloration in poplar. Although many genes might be involved in leaf coloration in poplar, the detailed functions of these anthocyanin-related genes should be further identified by experimental assays in the future.

\section{Methods}

\section{Identification of the PdeMYB gene family in P. deltoids}

The hidden Markov Model profile of MYB binding domain with accession number was obtained from the Pfam database (http://pfam.xfam.org/) [52], which was used to search candidate $P d e M Y B$ genes from $P$. deltoids genome with HMMER 3.1, with a cutoff value of 0.01 . The P. deltoids genome was downloaded from the Joint Genome Institute's Plant Genomics Portal (https://phytozome-next.jgi.doe.gov/), and HMMER 3.1 was downloaded from http://hmmer.org/download. html. In addition, $M Y B$ genes in Arabidopsis thaliana and rice were used to further identify candidate $P d e$ $M Y B$ genes in $P$. deltoids genome, which were downloaded from the Arabidopsis Information Resource (TAIR; http://www.arabidopsis.org/) and RGAP release 7 (http://rice.plantbiology.msu.edu/). The SMART and NCBI-CDD were used to confirm the acquired PdeMYB proteins in $P$. deltoids, the website of which were http://smart.embl-heidelberg.de and http://www.ncbi. nlm.nih.gov/Structure/cdd/wrpsb.cgi.

\section{Sequence analysis and structural characterization of $P$ deMYB genes in P. deltoids}

Based on the conserved domains and genome sequence of the PdeMYB genes, the exon-intron organization of the $P d e M Y B$ genes, including intron distribution patterns, phases, and intron-exon boundaries, was graphically displayed by the Gene Structure Display Server GSDS2.0 (http://gsds.cbi.pku.edu.cn/). The conserved motifs of the PdeMYB transcription factors were predicted using a MEME Suite analysis [53], and the software was downloaded from http://meme-suite.org/ tools/meme. The maximum number of motifs was set to identify 20 motifs, and the optimum width of motifs was set from 6 to 100 amino acids. 


\section{Chromosome distribution, gene duplication, and synteny of PdeMYB genes in P. deltoids}

The chromosome distribution of $P d e M Y B$ genes was obtained from the database of the $P$. deltoids genome (https://phytozome-next.jgi.doe.gov/), and the chromosomal locations of the PdeMYB genes were visualized using MapChart software [54]. Segmental duplication, tandem duplication, and synteny blocks of the orthologous $P d e M Y B$ genes between $P$. deltoids and Arabidopsis as well as $P$. deltoids and rice were evaluated using MCscanX (Multiple Collinearity Scan toolkit; http:// chibba.pgml.uga.edu/mcscan2/) [55]. The synonymous (Ks) and nonsynonymous (Ka) substitutions of PdeMYB genes were calculated using KaKs_Calculator 2.0 to estimate their duplication events [56], and circos v0.69 was used to graphically present the synteny blocks of the orthologous PdeMYB genes between $P$. deltoids and Arabidopsis, P. deltoids, and rice [57].

\section{Phylogenetic analysis and classification of PdeMYB proteins in $P$. deltoids}

Phylogenetic analysis was performed using the fulllength amino acid sequences of PdeMYB, OsMYB, and $A t M Y B$ proteins. An unrooted neighbor-joining phylogenetic tree was constructed through multiple sequence alignments of these $M Y B$ proteins using MEGA 7.0, and these $M Y B$ proteins were classified into different groups based on the topology of the phylogenetic tree. The parameters were as follows: pairwise deletion, Poisson model, and 1000 bootstrap replications [58]. The data matrices and resulting trees were deposited in TreeBase (http://purl.org/phylo/treebase/ phylows/study/TB2:S28602 and http://purl.org/phylo/ treebase/ phylows/ study/TB2:S28603), and the accession numbers are 28602 and 28603, respectively.

\section{Expression analyses of PdeMYB genes in Quanhong poplar (QHP) and Populus sp. Linn. 2025 (L2025) by RNA-seq}

To explore the expression pattern of $P d e M Y B$ genes in colored-leaf polar QHP and green leaf poplar L2025, the expression profiles of the putative PdeMYB genes in the leaves and buds of the QHP and L2025 were retrieved from previous research with $P d e M Y B$ gene IDs as the queries [59]. The transcript abundance of PdeMYB genes was calculated as fragments per kilobase of exon model per million mapped reads (FPKM). The $\log _{2}($ FPKM +1$)$ from the RNA-seq data were subjected to hierarchical clustering with Cluster 3.0, and the results were graphically displayed using Java TreeView [53].

\section{Expression analyses of PdeMYB genes between CHP and L2025 by qRT-PCR analysis}

Colored leaf poplar with bright red leaves, CHP, and green leaf poplar, L2025, were cultivated in the experimental field of the Nanjing Botanical Garden Mem. Sun Yat-Sen $\left(32^{\circ} 3^{\prime} \mathrm{N}, 118^{\circ} 49^{\prime} \mathrm{E}\right)$. To better identify the candidate $P d e M Y B$ genes associated with leaf color, the expression level of candidate $P d e M Y B$ genes in previous research that showed significant changes between QHP and L2025 were further evaluated. The leaves of CHP and L2025 were collected in August 2020, and the RNA was extracted using an RNA Aprep Pure Plant Kit (Tiangen, Beijing, China). The quality and concentration of each RNA sample was evaluated by gel electrophoresis and using a NanoDrop 2000 spectrophotometer (Thermo Fisher Scientific, Waltham, MA, USA), and the higher quality RNA with a 260/280 ratio of 1.8-2.1, $260 / 230$ ratio $\geq 2.0$, were stored at $-80{ }^{\circ} \mathrm{C}$ for further analyses. cDNA was synthesized using a ReverTra Ace qPCR RT kit (TOYOBO, Osaka, Japan). The expression levels of candidate PdeMYB genes in the leaves of CHP and L2025 were evaluated by qRT-PCR using an Applied Biosystems 7500 Real-Time PCR system (Applied Biosystems, Waltham, MA, USA). Gene-specific primers were designed according to the sequence of candidate PdeMYB genes (Additional file 6), and the ACTIN2 gene was used as a control gene [29]. The thermal cycling conditions were as follows: $95{ }^{\circ} \mathrm{C}$ for $2 \mathrm{~min}$, followed by 40 cycles of $95{ }^{\circ} \mathrm{C}$ for $5 \mathrm{~s}$, and products collected at 60 ${ }^{\circ} \mathrm{C}$ for $34 \mathrm{~s}$. The relative expression levels of genes were evaluated by the $2^{-\Delta \Delta C t}$ method [60], and analyzed using SPSS 17.0, with three biological replicates.

\section{Conclusions}

In the present study, $302 P d e M Y B$ transcription factors identified in $P$. deltoids. Genomic localization and paralogs of PdeMYB genes mapped 289 genes on 19 chromosomes, with collinearity relationships among genes. The conserved domain, gene structure, and evolutionary relationships of $P d e M Y B s$ were also established and analyzed. The expression levels of some PdeMYB genes were significantly different among different coloredleaf poplar varieties, which provided valuable clues for further functional characterization, in addition to providing candidate genes for the future improvement of leaf colorization in $P$. deltoids. 


\section{Supplementary Information}

The online version contains supplementary material available at https://doi. org/10.1186/s12870-021-03212-1.

Additional file 1. Information of PdeMYB genes identified in Populus deltoids.

Additional file 2. Segmentally and tandemly duplicated PdeMYB gene pairs.

Additional file 3. One-to-one orthologous relationships between Populus deltoids and Arabidopsis.

Additional file 4. One-to-one orthologous relationships between Populus deltoids and Oryza sativa.

Additional file 5.The candidate PdeMYB genes with their expression level in the leaves of QHP more than ten times than these in L2025 or specifically expressed in the leaves of QHP or L2025.

Additional file 6. Specific primers used in relative quantitative real-time RT-PCR.

\section{Acknowledgements}

We would like to thank Editage (www.editage.com) for English editing during the revision of this manuscript.

\section{Authors' contributions}

Conceptualization, ZW and WZ:; methodology, ZW and SX; investigation, LX and WT; writing - original draft preparation, ZW; writing - review and editing, ZW; visualization, WN and ZF supervision, ZW; project administration, WZ; funding acquisition, ZW. All authors have read and agreed to the published version of the manuscript.

\section{Funding}

This work was supported by the Jiangsu Agricultural Science and Technology Innovation Fund [CX(20)3039] and National Natural Science Foundation of China [31700585], which can be used to pay for reagents and article-processing charge.

\section{Availability of data and materials}

All data generated or analysed during this study are included in this published article and its supplementary information files. The phylogenetic matrix and trees are available in the TreeBASE repository for Figs. 1 and 2. The links for the phylogenetic matrix and trees of Figs. 1 and 2 are as follows: http://purl.org/ phylo/treebase/phylows/study/TB2:S28602 and http://purl.org/phylo/treeb ase/phylows/study/TB2: S28603, and the accession numbers are 28602 and 28603, respectively.

\section{Declarations}

\section{Ethics approval and consent to participate}

Not applicable.

\section{Consent for publication}

Not applicable.

\section{Competing interests}

The authors declare that they have no competing interests.

Received: 11 May 2021 Accepted: 6 September 2021

Published online: 23 September 2021

\section{References}

1. Lipsick JS. One billion years of Myb. Oncogene. 1996;13:223-35.

2. $\mathrm{Li} C, \mathrm{Ng}$ C, Fan LM. MYB transcription factors, active players in abiotic stress signaling. Environ Exp Bot. 2015;114:80-91.
3. Pabo CO, Sauer RT. Transcription factors: structural families and principles of DNA recognition. Annu Rev Biochem. 1992;61:1053-95.

4. Riechmann JL, Heard J, Martin G, Reuber L, Jiang CZ, Keddie J, et al. Arabidopsis transcription factors: genome-wide comparative analysis among eukaryotes. Science. 2000;290:2105-10.

5. Rosinski JA, Atchley WR. Molecular evolution of the Myb family of transcription factors: evidence for polyphyletic origin. J Mol Evol. 1998;46:74-83.

6. Jin H, Martin C. Multifunctionality and diversity within the plant MYBgene family. Plant Mol Biol. 1999;41:577-85.

7. Dubos C, Stracke R, Grotewold E, Weisshaar B, Martin C, Lepiniec L. MYB transcription factors in Arabidopsis. Trends Plant Sci. 2010;15:573-81.

8. Geethalakshmi S, Barathkumar S, Prabu G. The MYB transcription factor family genes in sugarcane (Saccharum sp.). Plant Mol. Biol Reprod. 2015;33:512-31.

9. Jiang C, Gu X, Peterson T. Identification of conserved gene structures and carboxy-terminal motifs in the Myb gene family of Arabidopsis and Oryza sativa L. ssp. indica. Genome Biol. 2004;5:R46.

10. Stracke R, Werber M, Weisshaar B. The R2R3-MYB gene family in Arabidopsis thaliana. Curr Opin Plant Biol. 2001:4:447-56.

11. Matus JT, Aquea F, Arce-Johnson P. Analysis of the grape MYB R2R3 subfamily reveals expanded wine quality-related clades and conserved gene structure organization across Vitis and Arabidopsis genomes. BMC Plant Biol. 2008;8:83.

12. Liu C, Wang X, Xu Y, Deng X, Xu Q. Genome-wide analysis of the R2R3MYB transcription factor gene family in sweet orange (Citrus sinensis). Mol Biol Rep. 2014;41:6769-85.

13. Cao ZH, Zhang SZ, Wang RK, Zhang RF, Hao YJ. Genome wide analysis of the apple MYB transcription factor family allows the identification of MdoMYB121 gene conferring abiotic stress tolerance in plants. PLoS One. 2013:8(7):e69955.

14. Wilkins O, Nahal H, Foong J, Provart NJ, Campbell MM. Expansion and diversification of the Populus R2R3-MYB family of transcription factors. Plant Physiol. 2009;149:981-93.

15. Martin C, Paz-Ares J. MYB transcription factors in plants. Trends Genet. 1997:13:67-73.

16. Paz-Ares J, Ghosal D, Wienand U, Peterson PA, Saedler $\mathrm{H}$. The regulatory $\mathrm{Cl}$ locus of Zea mays encodes a protein with homology to myb proto-oncogene products and with structural similarities to transcriptional activators EMBO J. 1987;6:3553-8.

17. Mandaokar A, Thines B, Shin B, Lange BM, Choi G, Koo YJ, et al. Transcriptional regulators of stamen development in Arabidopsis identified by transcriptional profiling. Plant J. 2006;46(6):984-1008.

18. Oppenheimer DG, Herman PL, Sivakumaran S, Esch J, Marks MD. A myb gene required for leaf trichome differentiation in Arabidopsis is expressed in stipules. Cell. 1991;67(3):483-93.

19. Wang D, Zhang Y, Zhang Z, Zhu J, Yu J. KaKs_Calculator 2.0:a toolkit incorporating gamma-series methods and sliding window strategies. Genom Proteom Bioinf. 2010;8:77-80.

20. Ambawat S, Sharma P, Yadav NR, Yadav RC. MYB transcription factor genes as regulators for plant responses: an overview. Physiol Mol Biol Plants. 2013;19:307-21.

21. Song S, Qi T, Huang H, Ren Q, Wu D, Chang C, et al. The Jasmonate-ZIM domain proteins interact with the R2R3-MYB transcription factors MYB21 and MYB24 to affect jasmonate-regulated stamen development in Arabidopsis. Plant Cell. 2011;23:1000-13.

22. Seo PJ, Park CM. MYB96-mediated abscisic acid signals induce pathogen resistance response by promoting salicylic acid biosynthesis in Arabidopsis. New Phytol. 2010;186:471-83.

23. Seo PJ, Lee SB, Suh MC, Park MJ, Go YS, Park CM. The MYB96 transcription factor regulates cuticular wax biosynthesis under drought conditions in Arabidopsis. Plant Cell. 2011:23:1138-52.

24. Chen N, Yang Q, Pan L, Chi X, Chen M, Hu D, et al. Identification of 30 MYB transcription factor genes and analysis of their expression during abiotic stress in peanut (Arachis hypogaea L.). Gene. 2014;533:332-45.

25. Espley RV, Hellens RP, Putterill J, Stevenson DE, Kutty-Amma S, Allan AC. Red colouration in apple fruit is due to the activity of the MYB transcription factor, MdMYB10. Plant J. 2007;49(3):414-27.

26. Deluc L, Bogs J, Walker AR, Ferrier T, Decendit A, Merillon JM, et al. The transcription factor $V V M Y B 5 b$ contributes to the regulation of 
anthocyanin and proanthocyanidin biosynthesis in developing grape berries. Plant Physiol. 2008;147(4):2041-53.

27. Lai Y, Li H, Yamagishi M. A review of target gene specificity of flavonoid R2R3-MYB transcription factors and a discussion of factors contributing to the target gene selectivity. Front Biol. 2013;8:577-98.

28. Aharoni A, De Vos CH, Wein M, Sun Z, Greco R, Kroon A, et al. The strawberry FaMYB1 transcription factor suppresses anthocyanin and flavonol accumulation in transgenic tobacco. Plant J. 2001;28(3):319-32.

29. Cho JS, Nguyen VP, Jeon HW, Kim MH, Eom SH, Lim YJ, et al. Overexpression of PtrMYB119, a R2R3-MYB transcription factor from Populus trichocarpa, promotes anthocyanin production in hybrid poplar. Tree Physiol. 2016;36:1162-76.

30. Wang $H H$, Wang $X Q$, Song $W M$, Bao $Y$, Jin $Y L$, Jiang CM, et al. PdMYB118, isolated from a red leaf mutant of Populus deltoids, is a new transcription factor regulating anthocyanin biosynthesis in poplar. Plant Cell Rep. 2019;38(8):10

31. Borevitz JO, Xia Y, Blount J, Dixon RA, Lamb C. Activation tagging identifies a conserved MYB regulator of phenylpropanoid biosynthesis. Plant Cell. 2000;12:2383-93.

32. Tohge T, Nishiyama Y, Hirai MY, Yano M, Nakajima J, Awazuhara M, et al. Transcriptome-wide identification of R2R3-MYB transcription factors in barley with their boron responsive expression analysis. Mol Gen Genomics. 2013;288:141-55.

33. An CH, Lee KW, Lee SH, Jeong YJ, Woo SJ, Chun H, et al. Heterologous expression of IbMYB1a by different promoters exhibits different patterns of anthocyanin accumulation in tobacco. Plant Physiol Biochem. 2015:89:1-10.

34. He XJ, Zhao XC, Gao LP, Shi XX, Dai XL, Liu YJ, et al. Isolation and characterization of key genes that promote flavonoid accumulation in purple-leaf tea (Camellia sinensis L.). Sci. Rep. 2018:8:130.

35. Wang LJ, Lu WX, Ran LY, Dou LW, Yao S, Hu J, et al. R2R3-MYB transcription factor MYB6 promotes anthocyanin and proanthocyanidin biosynthesis but inhibits secondary cell wall formation in Populus tomentosa. Plant J. 2019;99:733-51.

36. Hu QN, Yang L, Liu SD, Zhou LM, Wang XT, Wang W, et al. A repressor motif-containing poplar R3 MYB-like transcription factor regulates epidermal cell fate determination and anthocyanin biosynthesis in Arabidopsis. J Plant Biol. 2016;59:525-35.

37. Yoshida K, Ma DW, Constabel PC. The MYB182 protein down-regulates proanthocyanidin and anthocyanin biosynthesis in poplar by repressing both structural and regulatory flavonoid genes. Plant Physiol. 2015;167:693-710.

38. Wan SZ, Li CF, Ma XD, Luo KM. PtrMYB57 contributes to the negative regulation of anthocyanin and proanthocyanidin biosynthesis in poplar. Plant Cell Rep. 2017;36:1263-76.

39. Yang $L$, Zhao X, Ran LY, Li CF, Fan D, Luo KM. PtoMYB156 is involved in negative regulation of phenylpropanoid metabolism and secondary cell wall biosynthesis during wood formation in poplar. Sci Rep. 2017;7:41209.

40. Ma D, Reichelt M, Yoshida K, Gershenzon J, Constabel CP. Two R2R3-MYB proteins are broad repressors of flavonoid and phenylpropanoid metabolism in poplar. Plant J. 2018;96:949-65.

41. Chen YH, Yang XY, He K, Liu MH, Li JG, Gao ZF, et al. The MYB transcription factor superfamily of Arabidopsis: expression analysis and phylogenetic comparison with the rice MYB family, Plant Mol. Biol. 2006;60:107-24.

42. Smita S, Katiyar A, Chinnusamy V, Pandey DM, Bansal KC. Transcriptional regulatory network analysis of MYB transcription factor family genes in rice. Front Plant Sci. 2015;6:1157.

43. Katiyar A, Smita S, Lenka SK, Rajwanshi R, Chinnusamy V, Bansal KC. Genome-wide classification and expression analysis of MYB transcription factor families in rice and Arabidopsis. BMC Genomics. 2012;13:544.
44. Nuruzzaman M, Manimekalai R, Sharoni A, Satoh K, Kondoh H, Ooka H, et al. Genome-wide analysis of NAC transcription factor family in rice. Gene. 2010;465:30-44.

45. He QL, Jones DC, Li W, Xie FL, Ma J, Sun RR, et al. Genome-wide identification of R2R3-MYB genes and expression analyses during abiotic stress in Gossypium raimondii. Sci Rep. 2016;6:22980.

46. Schilling S, Kennedy A, Pan S, Jermiin LS, Melzer R. Genome-wide analysis of MIKC-type MADS-box genes in wheat: pervasive duplications, functional conservation and putative neofunctionalization. New Phytol. 2019. https://doi.org/10.1111/nph.16122.

47. Du H, Feng BR, Yang SS, Huang YB, Tang YX. The R2R3-MYB transcription factor gene family in maize. PLoS One. 2012;7. https://doi.org/10.1371/ journal.pone.0037463.

48. Du H, Wang YB, Xie Y, Liang Z, Jiang SJ, Zhang SS, et al. Genome-wide identification and evolutionary and expression analyses of MYB-related genes in land plants. DNA Res. 2013;20:437-48.

49. Singh UM, Chandra M, Shankhdhar SC, Kumar A. Transcriptome wide identification and validation of calcium sensor gene family in the developing spikes of finger millet genotypes for elucidating its role in grain calcium accumulation. PLoS One. 2014;9:e103963.

50. Wu C, Huang H, Yeh LSL, Barker WC. Protein family classification and functional annotation. Comput Biol Chem. 2003;27:37-47.

51. Stracke R, Holtgräwe D, Schneider J, Pucker B, Sörensen TR, Weisshaar B. Genome-wide identification and character isolation of R2R3-MYB genes in sugar beet (Beta vulgaris). BMC Plant Biol. 2014;14:249.

52. Finn RD, Coggill P, Eberhardt RY, Eddy SR, Mistry J, Mitchell AL, et al. The Pfam protein families database: towards a more sustainable future. Nucleic Acids Res. 2016;44:D279-85.

53. Bailey TL, Boden M, Buske FA, Frith M, Grant CE, Clementi L, et al. MEME SUITE: tools for motif discovery and searching. Nucleic Acids Res. 2009;37:W202-8.

54. Voorrips RE. MapChart: software for the graphical presentation of linkage maps and QTLs. J Hered. 2002;93:77-8.

55. Wang $Y$, Tang H, Debarry Jeremy D, Tan X, Li J, Wang X, et al. MCScanX: a toolkit for detection and evolutionary analysis of gene synteny and collinearity. Nucleic Acids Res. 2012;40:e49.

56. Wang S, Barron C, Schiefelbein J, Chen JG. Distinct relationships between GLABRA2 and single-repeat R3 MYB transcription factors in the regulation of trichome and root hair patterning in Arabidopsis. New Phytol. 2010;185:387-400.

57. Krzywinski M, Schein Jl. Circos: an information aesthetic for comparative genomics. Genome Res. 2009;19:1639-45.

58. Kumar S, Stecher G, Tamura K. MEGA7: molecular evolutionary genetics analysis version 7.0 for bigger datasets. Mol Biol Evol. 2016;33:1870.

59. Zhang F, Zhao JL, Wan XQ, Luo XM, Li W, Sun LX, et al. From green to red: large-scale transcriptome comparison of a bud sport in poplar (Populus deltoides). Acta Physiol Plant. 2016;38:244.

60. Livak KJ, Schmittgen TD. Analysis of relative gene expression data using real-time quantitative PCR and the 2(-Delta Delta C(T)) Method. Methods. 2001;25:402.

\section{Publisher's Note}

Springer Nature remains neutral with regard to jurisdictional claims in published maps and institutional affiliations. 